\title{
Re-irradiation of locoregional esophageal cancer recurrence following definitive chemoradiotherapy: A report of 6 cases
}

\author{
ATSUTO KATANO, HIDEOMI YAMASHITA and KEIICHI NAKAGAWA \\ Department of Radiology, University of Tokyo Hospital, Tokyo 113-8655, Japan
}

Received May 4, 2017; Accepted July 17, 2017

DOI: $10.3892 /$ mco.2017.1384

\begin{abstract}
There is currently no consensus on salvage therapy for recurrent esophageal cancer. Salvage surgery is a well-established option for attaining long-term survival; however, it is associated with a high risk of perioperative morbidity and mortality. A total of 6 patients who underwent re-irradiation for recurrence of locoregional esophageal cancer following definitive chemoradiotherapy were investigated. The median interval between initial radiotherapy and re-irradiation was 17.4 months (range, 6.4-59.2 months). Re-irradiation salvage therapy was mostly administered with concurrent chemotherapy, which consisted of several cycles of nedaplatin on day 1 and oral S-1 administration on days 1-14. The median survival after re-irradiation was 13.6 months (range, 1.9-33.3 months). A total of 3 patients who completed hyperfractionated radiation therapy survived for $>1$ year. One patient has had no signs of recurrence or late radiation toxicity for $>2$ years. Severe acute hematological adverse events (AEs) occurred in 3 patients, including 1 case of grade 4 leukopenia. One severe late AE occurred in 1 patient, who developed grade 3 dysphagia and became permanently dependent on percutaneous endoscopic gastrostomy tube feeding. Salvage radiotherapy is considered to be a good treatment option for inoperable locoregional recurrent esophageal cancer. The results of the present study demonstrated that re-irradiation, with or without chemotherapy, for recurrent esophageal carcinoma after definitive chemoradiotherapy was tolerable and yielded reasonably satisfactory results.
\end{abstract}

\section{Introduction}

The rate of locoregional recurrence after definitive chemoradiotherapy for esophageal carcinoma is $\sim 40-60 \%$ (1). A proportion of such patients are referred for salvage therapy.

Correspondence to: Dr Hideomi Yamashita, Department of Radiology, University of Tokyo Hospital, 7-3-1, Hongo, Bunkyo-ku, Tokyo 113-8655, Japan

E-mail: yamachan07291973@yahoo.co.jp

Key words: esophageal carcinoma, salvage therapy, radiotherapy, recurrence, squamous cell carcinoma
In general, a patient with multiple-site recurrence is treated by single-agent or combination chemotherapy. Platinum agents have become the key drugs in systemic esophageal cancer chemotherapy. For stage IV esophagogastric cancer, combination chemotherapy with epirubicin plus cisplatin and 5-fluorouracil (ECF regimen) has resulted in a 1-year survival rate of $37 \%$ and a median survival duration of 9.9 months (2). However, with this triplet drug regimen, complete remission or long-term survival is extremely rare.

Patients with a limited number of relapse sites, referred to as oligometastases or oligorecurrence (3), are considered to be suitable candidates for definitive local therapy with the goal of improving prognosis (4). For locoregionally recurrent lesions after definitive chemoradiotherapy, salvage surgery is the only established treatment strategy that may offer any chance of long-term survival (5). Swisher et al reported that salvage esophagectomy resulted in 5-year survival of $25 \%$ in selected patients (6). However, this procedure should be considered for carefully selected patients, due to the high incidence of morbidity and mortality (7).

Re-irradiation following previous definitive chemoradiotherapy is generally contraindicated, considering the radiation tolerance of the organs at risk, including the lung, trachea, esophagus and spinal cord. Since recovery from the effects of the initial radiation therapy occurs with time, the optimal prescription dose for re-irradiation depends on multiple determinants, including the initial treatment, histology of the primary tumor, and location of recurrence.

We herein report the results of 6 patients who underwent salvage re-irradiation therapy with tolerable toxicity for locally recurrent esophageal cancer.

\section{Case reports}

The consecutive cases of 6 patients who underwent salvage re-irradiation (5 of whom also received concurrent chemotherapy) for esophageal cancer recurrence following definitive chemoradiotherapy between January 2011 and June 2016 at the Department of Radiology of the University of Tokyo Hospital were retrospectively analysed. Written informed consent was obtained from all subjects, following careful explanation regarding the possibility of severe treatment-related toxicity. All enrolled cases satisfied the following eligibility criteria: i) A history of previous chemoradiotherapy for esophageal carcinoma; ii) limited number of locoregional recurrence sites, 
including supraclavicular lymph nodes that could be included within the radiotherapy fields; iii) no evidence of distant metastasis; iv) Karnofsky Performance Status (KPS) score at recurrence diagnosis $>70$; and v) $>6$ months between initial and salvage irradiation. The median survival time (MST) was measured from the first day of re-irradiation. Clinical stage was determined according to the 7th edition of the American Joint Committee on Cancer TNM staging system (8). Treatment response was assessed according to the Response Evaluation Criteria in Solid Tumors guidelines, version 1.1 (https://ctep.cancer.gov/protocoldevelopment/docs/recist_guideline .pdf). Acute or late adverse events (AEs) were graded using the Common Terminology Criteria for Adverse Events, version 4.0 (https://evs.nci.nih.gov/ftp1/CTCAE/CTCAE_4.03_2010-06-14_ QuickReference_5x7.pdf).

All radiotherapy was delivered using a 3-dimensional conformal radiation therapy technique with 6 or $10 \mathrm{MV}$ photon energies. The clinical target volume (CTV) of radiotherapy was the macroscopic tumor and enlarged lymph nodes, if any, surrounded by sufficient proximal and distal margins. The CTV was delineated on planning computed tomography (CT) images, with reference to positron emission tomography (PET) image data, if available. The CTV to the planning target volume (PTV) margin was $0.5-1.5 \mathrm{~cm}$. As this was re-irradiation planning, the multiple beam arrangement technique was used to avoid irradiation of the spinal cord. Follow-up examinations included a physical examination and assessment of laboratory values and imaging examinations every 1-3 months.

In our institution, concurrent chemotherapy consists of nedaplatin and oral S-1 administration or intravenous 5-fluorouracil infusion. S-1 is an oral prodrug of 5-fluorouracil, which can be administered in an outpatient setting, and nedaplatin produces less nausea, vomiting and nephrotoxicity compared with other platinum-containing drugs (9). Tsuda et al evaluated the feasibility of S-1 and nedaplatin in combination with radiotherapy, and reported that the toxicity was tolerable in esophageal cancer treatment (10). Yamashita et al also reported the efficacy and feasibility of nedaplatin plus S-1 in definitive or salvage concurrent chemoradiotherapy for early, advanced and relapsed esophageal cancer (11).

All the patients were male and the median age at the time of initial radiation treatment was 65 years (range, 52-68 years). The patient characteristics and initial treatment modalities are summarized in Table I. Patient D suffered progressive disease after the third cycle of chemotherapy in initial treatment with the appearance of a new out-of-field lesion in the esophagus; he underwent salvage esophagectomy with a gastric conduit reconstruction. Patient $\mathrm{E}$ had undergone endoscopic submucosal dissection (ESD) 6 months prior to chemoradiotherapy. The ESD result had revealed invasion of the second submucosal layer, but the patient declined adjuvant therapy at that time. Recurrence in a superior mediastinal lymph node developed 6 months later, and the patient was clinically staged as rTxN1M0; he then underwent definitive chemoradiotherapy, resulting in complete response.

The median time interval between initial radiotherapy and re-irradiation was 17.4 months (range, 6.4-59.2 months). A whole-body PET scan was available for all but 1 patient (patient F), confirming the absence of distant metastatic

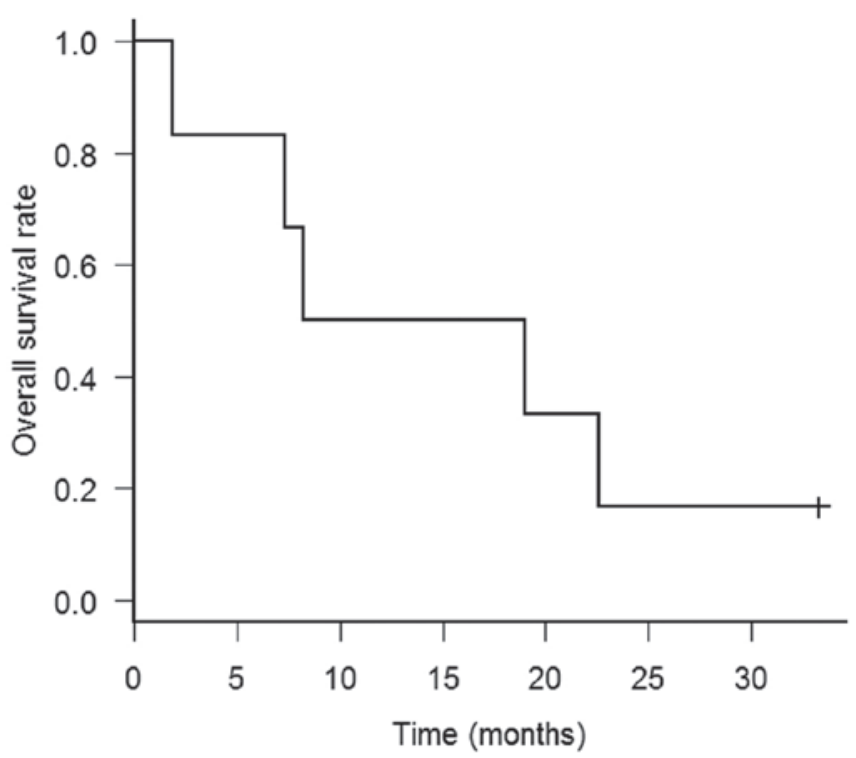

Figure 1. Kaplan-Meier curves for overall survival. A vertical bar indicates a censored case.

lesions and guiding target volume delineation. The re-irradiation delivered dose ranged from 30 to $50.4 \mathrm{~Gy}$, with fractionation of 1.8-2.0 Gy per day or 1.2-1.5 Gy twice daily. A total of 5 patients underwent concurrent chemotherapy with nedaplatin and oral S-1 administration with radiotherapy, and the remaining patient underwent radiotherapy alone. The other characteristics at recurrence are summarized in Table II. Patient E developed recurrence in a superior mediastinal lymph node after the first definitive chemoradiotherapy and underwent salvage surgery; however, the surgery was limited by lesions involving arteries, and he was referred to our department for re-irradiation as salvage therapy. Patient F had his fractionation changed from twice daily to once daily after lymphadenopathy was noted after he had received $18 \mathrm{~Gy}$.

Severe (grade 3-5) acute hematological AEs occurred in 3 patients, including 1 case of grade 4 leukopenia. There were no acute non-hematological AEs of grade $\geq 3$. No radiation myelitis, esophageal perforation, or fissure occurred. One patient developed grade 3 dysphagia and became permanently dependent on a percutaneous endoscopic gastrostomy tube feeding $\sim 6$ months after the start of re-irradiation. The same patient also developed grade 2 hypothyroidism, requiring levothyroxine starting 3 months after irradiation to the time of death.

The MST after re-irradiation was 13.6 months (range, 1.9-33.3 months) (Fig. 1). Only 1 patient (patient C) remained alive at the time of the analysis. The longest disease-free survival from re-irradiation to second recurrence was 33.3 months. The patient underwent salvage chemotherapy and was alive without any evidence of disease. Patient B also remained disease-free for $>1$ year after re-irradiation. Fifteen months after the starting date of re-irradiation, the patient experienced hoarseness and the CT scan revealed superior mediastinal lymphadenopathy and vertebral metastasis and he underwent salvage chemotherapy consisting of oral S-1 administration. 


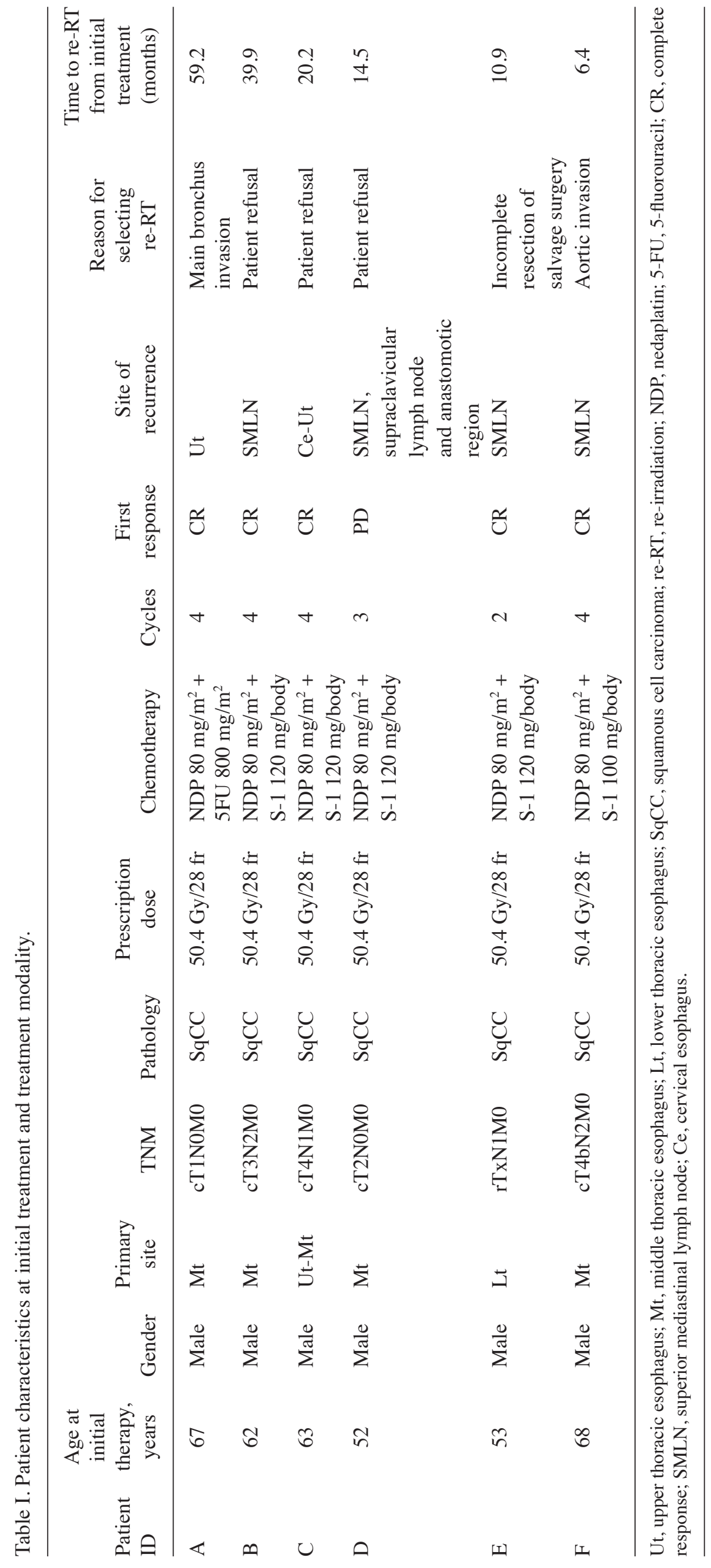




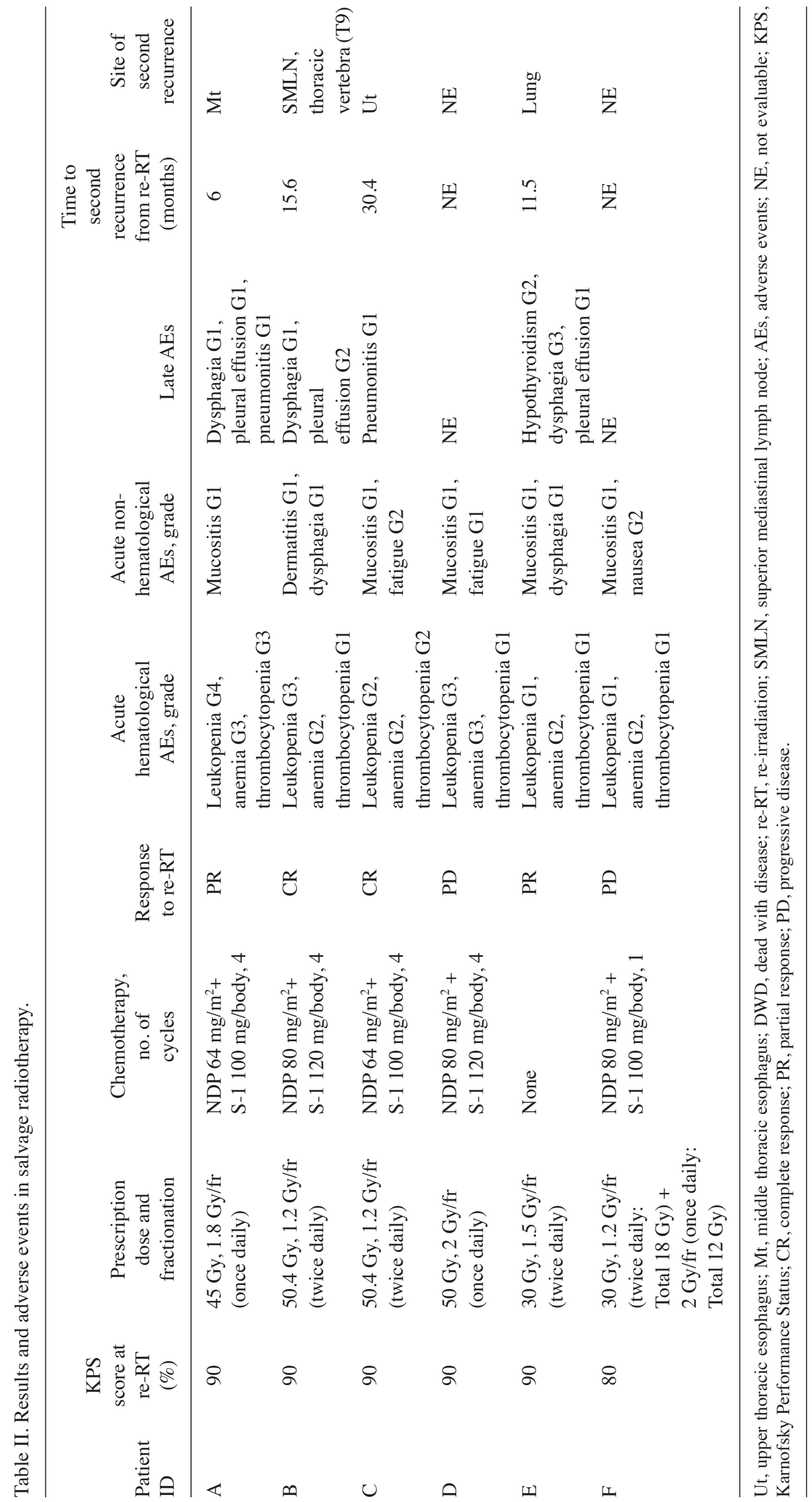


Patients D and F exhibited rapid progression of recurrent disease soon after re-irradiation. Patients A and E experienced temporary responses to salvage chemoradiotherapy; however, disease recurrence occurred in the middle thoracic esophagus and lung, respectively.

\section{Discussion}

Esophageal cancer is the seventh most common cause of cancer-related mortality in Japan, with an estimated 11,746 deaths in 2008 (12). Re-irradiation salvage therapy, with or without chemotherapy, for recurrent esophageal carcinoma after definitive chemoradiotherapy yielded reasonable results in this small group of patients, extending MST from the expected 9.9 months (2) to 13.6 months. Although treatment toxicity is one of the issues of most concern at re-irradiation, particularly late AEs, there was no lethal treatment-related toxicity in the present study, and only 1 of 6 patients $(16.7 \%)$ developed grade 3 late AEs.

Zhou et al also reported on the efficacy and feasibility of salvage radiotherapy in patients with locally recurrent esophageal cancer after definitive chemoradiotherapy (13). The 3-year overall survival rate was $21.8 \%$. Radiation pneumonitis grade $\geq 3$ was observed in $5.45 \%$, and esophageal fistula/perforation was observed in $20.0 \%$ of the cases. Fakhrian et al reported on 54 patients with recurrent esophageal carcinoma undergoing salvage radiotherapy (14). Median survival time was 12 months (95\% confidence interval: 7-17 months) and toxicity was in an acceptable range. Kim et al described 10 patients who underwent re-irradiation of recurrent esophageal cancer after primary definitive radiotherapy (14); 2 patients had a complete response, but 3 succumbed to esophageal perforation within 2 months of completion of re-irradiation.

Salvage surgery is a treatment modality for locoregional recurrence of esophageal cancer. The incidence of adverse events of salvage surgery following definitive chemoradiotherapy is higher compared with that of initial surgery. Tachimori et al reported that patients who underwent salvage esophagectomy, compared with those who underwent esophagectomy without preoperative therapy, experienced increased anastomotic leak rates ( 31 vs. $25 \%$, respectively), respiratory complication rates (31 vs. $20 \%$, respectively), and hospital mortality rates ( 8 vs. $2 \%$, respectively) (15). Chen et al compared salvage chemoradiotherapy with surgery for recurrent esophageal squamous cell carcinoma following definitive radiotherapy (16); they did not observe a statistically significant survival difference $(\mathrm{P}=0.697)$, but the frequency of complications, including esophageal fistula/perforation, was higher $(19.0 \%)$ in the salvage chemoradiotherapy group.

In the results presented herein, 3 patients who completed hyperfractionated re-irradiation therapy remained alive 1 year after salvage treatment. Hyperfractionated radiation has been considered to improve locoregional control rates without increasing late toxicity in head and neck cancer (17). In patients with recurrent rectal carcinoma, high doses of hyperfractionated radiation may be delivered with acceptable risk, without prohibitive long-term side effects (18). Hyperfractionated re-irradiation has also been used to successfully manage locally recurrent lung cancer (19) and head and neck cancers (20). Moreover, hyperfractionated radiotherapy resulted in favorable outcomes for Japanese patients with head and neck carcinoma $(21,22)$.

In conclusion, although the outcome of re-irradiation for recurrent esophageal cancer was poor, some patients obtained long-term disease control without any severe symptoms. Re-irradiation may be a useful modality as salvage therapy for patients who are not surgical candidates. Re-irradiation-related AEs were compatible with those of previous reports. However, the sample size of this study was very limited, and confirmation with a larger number of patients and a longer follow-up period is required.

\section{References}

1. Pennathur A, Gibson MK, Jobe BA and Luketich JD: Oesophageal carcinoma. Lancet 381: 400-412, 2013.

2. Cunningham D, Starling N, Rao S, Iveson T, Nicolson M, Coxon F, Middleton G, Daniel F, Oates J and Norman AR; Upper Gastrointestinal Clinical Studies Group of the National Cancer Research Institute of the United Kingdom: Capecitabine and oxaliplatin for advanced esophagogastric cancer. N Engl J Med 358: 36-46, 2008.

3. Niibe Y and Chang JY: Novel insights of oligometastases and oligo-recurrence and review of the literature. Pulm Med 2012: 261096, 2012.

4. Niibe Y and Hayakawa K: Oligometastases and oligo-recurrence: The new era of cancer therapy. Jpn J Clin Oncol 40: 107-111, 2010.

5. Takeuchi H, Saikawa Y, Oyama T, Ozawa S, Suda K, Wada N, Takahashi T, Nakamura R, Shigematsu N, Ando N, et al: Factors influencing the long-term survival in patients with esophageal cancer who underwent esophagectomy after chemoradiotherapy. World J Surg 34: 277-284, 2010.

6. Swisher SG, Wynn P, Putnam JB, Mosheim MB, Correa AM, Komaki RR, Ajani JA, Smythe WR, Vaporciyan AA, Roth JA and Walsh GL: Salvage esophagectomy for recurrent tumors after definitive chemotherapy and radiotherapy. J Thorac Cardiovasc Surg 123: 175-183, 2002.

7. D'Journo XB and Thomas PA: Current management of esophageal cancer. J Thorac Dis 6 (Suppl 2): 253-264, 2014.

8. Ota K: Nedaplatin. Gan To Kagaku Ryoho 23: 379-387, 1996 (In Japanese).

9. Tsuda $T$, Inaba $H$, Miyazaki A, Izawa N, Hirakawa M, Watanabe Y, Kitajima S, Hoshikawa Y, Gomi H, Kimura M and Itoh F: Prospective study of definitive chemoradiotherapy with S-1 and nedaplatin in patients with stage II/III (non-T4) esophageal cancer. Esophagus 8: 45-51, 2011.

10. Yamashita H, Haga A, Takenaka R, Kiritoshi T, Okuma K, Ohtomo K and Nakagawa K: Efficacy and feasibility of ambulatory treatment-based monthly nedaplatin plus S-1 in definitive or salvage concurrent chemoradiotherapy for early, advanced, and relapsed esophageal cancer. Radiat Oncol 11: 4, 2016.

11. Lin Y, Totsuka Y, He Y, Kikuchi S, Qiao Y, Ueda J, Wei W, Inoue $\mathrm{M}$ and Tanaka $\mathrm{H}$ : Epidemiology of esophageal cancer in Japan and China. J Epidemiol 23: 233-242, 2013.

12. Zhou ZG, Zhen CJ, Bai WW, Zhang P, Qiao XY, Liang JL, Gao XS and Wang SS: Salvage radiotherapy in patients with local recurrent esophageal cancer after radical radiochemotherapy. Radiat Oncol 10: 54, 2015.

13. Fakhrian K, Gamisch N, Schuster T, Thamm R, Molls M and Geinitz H: Salvage radiotherapy in patients with recurrent esophageal carcinoma. Strahlenther Onkol 188: 136-142, 2012.

14. Kim YS, Lee CG, Kim KH, Kim T, Lee J, Cho Y and Koom WS: Re-irradiation of recurrent esophageal cancer after primary definitive radiotherapy. Radiat Oncol J 30: 182-188, 2012.

15. Tachimori Y, Kanamori N, Uemura N, Hokamura N, Igaki H and Kato H: Salvage esophagectomy after high-dose chemoradiotherapy for esophageal squamous cell carcinoma. J Thorac Cardiovasc Surg 137: 49-54, 2009.

16. Chen Y, Lu Y, Wang Y, Yang H, Xia Y, Chen M, Song H, Li T, Li D, Wang J, et al: Comparison of salvage chemoradiation versus salvage surgery for recurrent esophageal squamous cell carcinoma after definitive radiochemotherapy or radiotherapy alone. Dis Esophagus 27: 134-140, 2014. 
17. Beitler JJ, Zhang Q, Fu KK, Trotti A, Spencer SA, Jones CU, Garden AS, Shenouda G, Harris J and Ang KK: Final results of local-regional control and late toxicity of RTOG 9003: A randomized trial of altered fractionation radiation for locally advanced head and neck cancer. Int J Radiat Oncol Biol Phys 89: 13-20, 2014

18. Mohiuddin M, Marks G and Marks J: Long-term results of reirradiation for patients with recurrent rectal carcinoma. Cancer 95: 1144-1150, 2002.

19. Okamoto Y, Murakami M, Yoden E, Sasaki R, Okuno Y, Nakajima $T$ and Kuroda Y: Reirradiation for locally recurrent lung cancer previously treated with radiation therapy. Int J Radiat Oncol Biol Phys 52: 390-396, 2002.

20. Watkins JM, Shirai KS, Wahlquist AE, Stuart RK, Chaudhary UB, Garrett-Mayer E, Day TA, Gillespie MB and Sharma AK: Toxicity and survival outcomes of hyperfractionated split-course reirradiation and daily concurrent chemotherapy in locoregionally recurrent, previously irradiated head and neck cancers. Head Neck 31: 493-502, 2009.
21. Niibe Y, Karasawa K, Mitsuhashi $T$ and Tanaka Y: Hyperfractionated radiation therapy for hypopharyngeal carcinoma compared with conventional radiation therapy: Local control, laryngeal preservation and overall survival. Jpn J Clin Oncol 33: 450-455, 2003.

22. Niibe Y, Karasawa K, Mitsuhashi T, Miyashita H and Tanaka Y: Hyperfractionated radiation therapy for oropharyngeal carcinoma in a Japanese population. Jpn J Clin Oncol 34: 312-315, 2004 